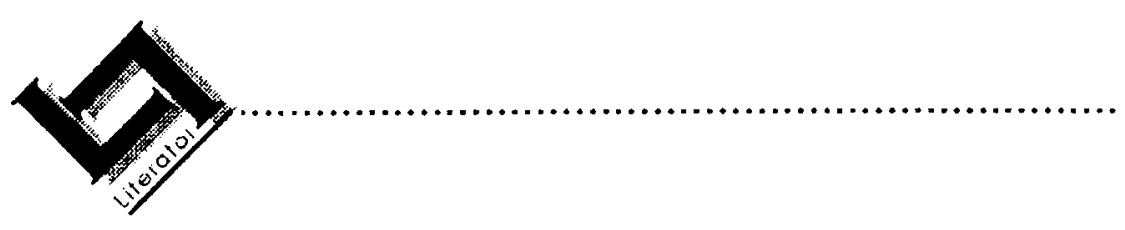

\title{
Assia Djebar, de l'écriture au cinéma
}

\author{
Serge-Dominique Ménager \\ French Section \\ School of Language, Culture and Communication \\ Natal University \\ PIETERMARITZBURG \\ E-pos: menager@nu.ac.za
}

\begin{abstract}
Assia Djebar, from writing to filming

In 1978 Assia Djebar was already a well established Francophone Algerian woman writer. It was during that year that her first film La nouba des femmes du mont Chenoua was shown in Algerian cinemas. This first attempt was followed by a second film La Zerda ou les chants de l'oubli. These concurrent creative processes show how, for Djebar, writing and filming are two closely linked activities.
\end{abstract}

\section{Introduction}

Dans le domaine de la littérature française, les écrivains ayant tenté parallèlement à leur œuvre écrite une carrière cinématographique ne sont pas rares. Avec des bonheurs divers (Espoir, 1939 l'unique réalisation d'André Malraux, ou les réelles réussites de Cocteau et de Duras), nombreux furent les créateurs qui trouvèrent dans l'aventure du film une voie d'expression et de création aussi forte et satisfaisante que celle de l'écriture. Les relations entre écriture et film ont, dés l'invention du cinéma, occupé une place centrale dans les travaux des théoriciens. La division initiale entre cinéma de fiction et cinéma documentaire est représentée de manière symbolique par deux courants opposés. D'une part les fréres Lumière 1 et les opérateurs Gaumont, envoyés aux quatre coins du monde pour en rapporter des images inédites à des spectateurs assoiffés d'exotisme, posèrent les bases de l'école réaliste et d'autre

1 Auguste (1862-1954) et Louis (1864-1948) Lumière, inventeurs du Cinèmatographe pour lequel ils tournèrent de nombreux films. 
part, Georges Mélies 2 établit les fondements du cinéma de fiction qui tout naturellement puisa dans les innombrables ressources que lui offrait la littérature. L'évidence de cette filiation du cinéma à la littérature est comme matérialisée par la fondation de la compagnie cinématographique américaine Paramount développée à l'origine uniquement autour de l'énorme succès des adaptations filmées de pièces de théàtre interprétées à l'écran, en version muette, par la célèbre tragédienne Sarah Bernhardt. La théorisation des rapports entre écriture littéraire et écriture cinématographique atteindra son apogée dans les recherches du cinéaste-théoricien Alexandre Astruc (1923-1993) qui développera dans les années soixante sa théorie de la caméra-stylo.

\section{Assia Djebar première femme-cinéaste maghrěbine}

Pour ce qui concerne la littérature africaine, les exemples d'écrivainscinéastes sont plus rares mais on compte de brillantes réussites. Le double cheminement littéraire et filmique de Sembène Osmane constitue un succès exemplaire mais cette entreprise duelle s'avère plus rare dans le cadre de la littérature maghrébine d'expression française et Assia Djebar constitue une exception tout-à-fait unique. Elle est en effet le seul écrivain-cinéaste. Elle a été la première femme cinéaste (la marocaine Farida Benlyazid ne tournera son premier long métrage qu'en 1988 soit dix ans plus tard que Djebar) et Hafsa Zinai-Koudil (unique autre femme cinéaste recensée par les auteurs du Dictionnaire des cinéastes du Maghreb (1996) sous la rubrique "Algérie" ne tournera un film qu'en 1993. Seule la tunisienne Selma Baccar peut prétendre à la mème ancienneté dans la profession. ${ }^{3}$

Lorsqu'elle entreprend le tournage de sa première œuvre cinématographique, La nouba des femmes du mont Chenoua en 1978, Djebar est déjà un écrivain de renommée: elle a publié entre autres Les enfants du nouveau monde (1962) et Les alouettes naïves (1967). C'est déjà un auteur de poids et rien dans son œuvre écrite ne laisse supposer un virage vers le cinéma. Pourtant, cette expérience n'est pas arrivée par hasard - dans le contexte de l'Algérie de 1977, année du tournage, faire un film n'est pas une mince affaire et reste principalement une tàche

2 Georges Méliés (1861-1938), pionnier du spectacle cinématographique, constructeur des premiers studios, il réalisa plus de 500 petits films.

3 Aprés une assez longue période d'assistanat à la réalisation qui suivit le tournage de deux courts-métrages (1968), Selma Baccar réalisa le premier long métrage tunisien dirigé par une femme Fatma 75 en 1978 qui fut censuré par le gouvernement. Ce ne fut qu'en 1995 que la réalisatrice tunisienne entreprit son second long métrage, La danse du feu / Habiba Msika. 
d'homme. 1978 verra la sortie de six films, tous masculins excepté celui qui nous intéresse. L'ONCIC (Office National du Commerce et de I'Industrie Cinématographiques) est en pleine crise et se relève difficilement d'un scandale lié à son président Mohammed Lakhdar Amina, accusé d'avoir mobilisé au profit de son énorme production Chronique des années de braise - qui remportera la palme d'or à Cannes en 1975 - tout l'argent de l'organisme qui aurait pu être utilisé au financement d'autres productions plus modestes. Les fonds sont rares et Djebar ne peut compter que sur son nom de romanciêre pour monter son projet de film. Celui-ci est en fait produit par la RTA (Radio Télévision Algérienne) puis distribué en salles par l'ONCIC. L'année du tournage est aussi celle qui suit la mort du Président Houari Boumédienne, celle donc d'une certaine instabilité, due à i'avenir imprécis du FLN.

Si rien ne signale dans l'œuvre écrite l'intérêt pour le genre filmé, il faut cependant premiérement noter que Djebar n'a plus écrit depuis 1967, soit dix ans au moment du tournage. On peut donc envisager un possible blocage de l'écrit qui essayerait peut-être de se résoudre par la production d'images. Deuxiémement, durant ces mois de tournage, l'écrivain a cependant un projet en tête qu'elle structure peu à peu, un recueil de nouvelles Femmes d'Alger dans leur appartement.

\section{Influences de l'avant-garde}

La vision de La nouba des femmes du mont Chenoua nous découvre une connaissance approfondie du cinéma international de l'époque. Djebar ne vient pas au film en complète innocence, c'est une cinéphile avertie et dans ses choix, ses partis-pris esthétiques, se lit un goût prononcé pour le cinéma d'avant-garde de l'époque. On trouve dans cette première œuvre des références très claires à l'état du cinéma durant ces années 70 qui ont déjà vu la mort de la nouvelle vague française et l'évolution de son leader, Jean-Luc Godard, vers une œuvre politiquement engagée. Godard a renoncé au cinéma d'auteur et signe ses films sous le nom "de Groupe Dziga Vertov"4. Ses réalisations tentent, selon une ligne de pensée maoïste en vogue à l'époque de répondre à une question majeure, "des films pour qui et contre qui?" Des œuvres comme Vent d'est (1970) et plus tard Tout va bien (1972) ont développé une nouvelle utilisation du son qui reste une des innovations majeures du cinéma de ces années et dont la théorisation est

4 Dziga Vertov (1885-1954), cinéaste soviétique dont les principes de tournage et de montage influencérent entre autres les mouvements du cinéma-vérité et du néoréalisme italien. L'homme à la caméra (1930) est son film-manifeste le mieux connu. 
développée dans la revue Les cahiers du cinéma. II s'agit avant tout d'une approche politique de la pratique du cinéma structurée selon le principe d'une dialectique marxiste dont les deux composants sont le son et l'image. Celle-ci a été analysée et développée par des théoriciens comme Pascal Bonitzer, Serge Daney, réflexion ensuite élargie par Jean-Pierre Oudart dans un texte capital pour la compréhension de ces expériences, "La suture" 5 Pour résumer cette réflexion on peut dire que certains cinéastes de cette époque tentent de renverser la primauté longtemps établie des images sur la bande son. C'est l'époque des manifestes, l'écran se vide, l'image s'y étire en plan-séquence tandis que le haut-parleur déverse des flots d'une richesse ininterrompue et parfois difficile à saisir puisque le son direct souvent brouillon est préféré au son reconstitué et artificiel du studio6.

Parallèlement à Godard, une autre cinéaste se distingue à la même époque. Bien qu'employant les mêmes techniques, celles-ci sont mises au service de son inspiration personnelle. Marguerite Duras, après des débuts presque "classiques" avec La Musica en 1969, réalise en effet deux œuvres majeures Détruire dit-elle (1969) mais surtout India Song, poème-manifeste d'un cinéma entièrement mis au service de son auteur. India Song est sorti en France en 1975 et pour qui a vu le cinéma de Djebar, il est évident que la réalisation de Duras a eu une influence majeure sur ses deux films et par extension sur son travail écrit.

\section{La nouba des femmes du mont Chenoua}

Pour mieux comprendre cette influence, une description rapide de ce premier film s'impose. Comme chez Duras (faut-il insister sur les titres o combien significatifs: Moderato Cantabile, La musica, India Song), la musique joue un rôle capital dans la conception même de l'œuvre, dans sa structure. La nouba des femmes du mont Chenoua ouvre sur une dédicace révélatrice: "Ce film en forme de nouba est dédié à titre posthume au musicien Bela Bartok venu en 1913 dans une Algérie quasiment muette étudier la musique populaire ... " (Djebar, 1978). Tout au long de l'œuvre, la bande son associera musique classique européenne (celle de Bartok, en l'occurrence) et musique locale. La

5 "La suture", Cahiers du Cinéma, No 212, mai 1969, p. 50. Consulter aussi le livre de Serge Daney, L'Exercice a été profitable, Monsieur, aux Éditions POL, 1993.

6 Pour de plus amples informations sur le cinéma international d'avant-garde de cette période lire le numéro hors-série des Cahiers du cinéma, "Cinéma 68". M1089,. Paris : Edition de l'Etoile. Lire aussi les livres de Benjamin Lambert, Défense d'interdire, Almanach (nostalgique) de mai 1968 et de Jacques Patoz et Jean-Michel Saint-Ouen, Révoltes tous deux parus aux éditions Méréal, Paris, 1998. 
dédicace conclut: "La nouba est aussi une sorte de symphonie en musique classique dite 'andalouse' avec des mouvements rythmiques déterminés" (1978).

Les éléments musicaux ne se confinent cependant pas à ces deux pôles: Djebar y ajoute encore la musique populaire algérienne qui intervient en contrepoint. Les paroles des chansons utilisées semblent apporter un commentaire au fil de l'histoire racontée en particulier à la fin du film 7 où elles convoient un message politique:

Mon chant parle toujours de liberté j'intercède pour les femmes martyres et que les autres ne soient plus opprimées ... les femmes ne retourneront plus dans l'ombre ... au temps de la servitude on a justifié le voile mais maintenant commence le jour de la liberté (Djebar, 1978).

Dernier élément, le film comporte une séquence où apparaît un groupe de musiciens (c'est en fait le groupe Ichenoui qui dans les années 70 chantait en patois berbére local, des chansons inspirées du folklore de la région du Chenoua - autour de Tipaza et Cherchell). Ils chantent le long de la mer, à côté de la demeure de l'héroïne.

La structure même du film est agencée sur une forme musicale : les séquences s'enchaïnent au long de titres renvoyant à autant de mouvements: vient d'abord la Touchia, ouverture, puis l'Istikar, le prélude, que continue le Meceder, adagio puis suivent Btaihi, Deroj, Nesraf, et enfin le final, Khlass. Autant de mouvements que de femmes mises en scène.

Dans son utilisation de la voix off Djebar est donc inspirée par Duras mais elle ne se montre pas aussi radicale que cette derniêre. On retrouve bien l'idée d'une polyphonie comme chez Godard ou dans le hors-champ sonore de India Song mais il s'agit avant tout d'un long commentaire intérieur de l'héroïne réfléchissant sur sa condition personnelle et revêtant la forme de la voix narratrice traditionnelle. Le message reste toujours $100 \%$ audible, aucune tentative de brouillage par superposition des sons n'est ici tentée.

Voici comment le Dictionnaire des cinéastes du Maghreb de Roy Armes résume l'intrigue du film:

7 Les paroles de la chanson de la fin du film ont été écrites par Djebbar elle-mème. 
Architecte, Leila retourne dans la maison familiale pour savoir dans quelles conditions son frère est mort pendant la guerre de Libération. Son itinéraire se mêle aux souvenirs de six vieilles femmes qui ont vécu cette guerre, et aux légendes et batailles ancestrales de la région qu'elle parcourt (Armes, 1996:32).

Au visionnage, l'histoire est à la fois moins claire et moins directement saisissable. Ce manque de clarté est peut-être dû à l'ambition de Djebar qui ne se limite pas à cette multiplication des personnages. En effet, les six femmes en question se confondent et se rejoignent toutes en une femme unique, la maquisarde Yasmina Oudaï dite Zoulikha, morte aux mains des soldats français durant la guerre d'indépendance - le film lui est aussi dédié. Et s'il y a brouillage de l'œuvre, c'est plus au niveau narratif qu'au niveau sonore. L'identité même de l'héroïne semble se dissoudre (la modernité des années 70 est encore très soumise aux expériences du Nouveau Roman). Leila paraît d'abord n'être qu'un personnage extérieur aux témoignages recueillis par elle, puis elle est assimilée à la fille de la martyre, et finalement à la martyre elle-même: "C'était moi, c'était elle" (Djebar, 1978) s'exclame soudain la jeune fille.

Cette complexité se renforce encore d'un télescopage entre le récit présent et une évocation de la révolte nationaliste de 1871 dans la région du Chenoua (Sidi Malek Sahraoui). Les deux narrations s'entremêlent, court-circuitées par certaines légendes racontées (celle du Saint du Chenoua, par exemple). On passe donc de séquences réalistes tournées dans le style du reportage (interviews des paysannes) à des segments d'inspiration poético-lyrique et historique.

Le film constitue ainsi un kaléidoscope parfois malaisé à suivre. La nouba des femmes du mont Chenoua pose en effet les jalons d'un véritable renouvellement de l'œuvre à venir de Djebar. On peut penser que le film ne parvient sans doute pas à réaliser les ambitions de son metteur en scène par manque de moyens financiers d'une part, mais surtout par le jeu peu convaincant des acteurs. II n'en reste cependant pas moins vrai qu'il constitue une étape essentielle pour l'auteur que viendra compléter le film suivant. Celui-ci raconte l'histoire de "l'entrée du pays dans la nuit coloniale" (Djebar, 1982) en passant par les deux guerres mondiales, et en évoquant des personnages comme l'Emir Abdelkader, Abdelkrim el Khattabi ainsi que la répression des révoltes anti coloniales par l'armée française. L'œuvre se termine sur le débarquement américain à Casablanca, en 1942. 


\section{La zerda ou les chants de l'oubli}

Une analyse de La Zerda ou les chants de l'oubli révèle d'abord une frappante similarité entre les deux œuvres: le titre même est construit autour d'un thème musical, nouba dans un cas, zerda dans l'autre. La division du film se réfêre encore directement à la musique: 5 parties se succèdent dont 4 portent le nom d'un chant, "Chant de l'insoumission", "Chant de l'intransigeance", "Chant de l'insolation", "Chant de l'émigration". Le procédé de la voix off se retrouve, mais boursoufflé au point d'envahir toute la bande son qui s'en trouve démultipliée. II ne s'agit plus de l'introspection d'une héroïne mais d'une multiplicité de voix chantant à l'unisson et se répondant ou s'affrontant les unes les autres. Comme dans son premier film, Djebar ouvre son récit par un avertissement qui n'est pas sans rappeler le précédent :

Dans un Maghreb totalement soumis et réduit au silence, photographes et cinéastes ont afflué pour nous prendre en images. La 'Zerda' est cette 'fête' moribonde qu'ils prétendent saisir de nous. A partir du hors champ de leur regard qui fusille nous avons tenté de faire lever d'autres images, lambeau d'un quotidien méprisé (Djebar, 1982).

La cinéaste met l'accent sur ce qui différencie radicalement le second film du premier, l'image. Cette fois-ci elle ne travaille plus sur un matériau de sa création, elle met bout à bout des bobines d'archives du cinéma muet puis parlant, filmées par les Européens. Elle semble avoir échappé au tiraillement entre le documentaire et la fiction qui faisait l'originalité de La nouba des femmes du mont Chenoua. Partant d'un matériau brut donné qu'elle personnalise d'abord par le montage, elle peut enfin donner toute son ampleur à ses expériences sur le son qui devient ici le moteur dramatique et émotionnel de son travail. On retrouve l'écho de ce projet dans la suite du texte d'introduction: "Surtout, derrière le voile de cette réalité exposée se sont réveillées des voix anonymes, recueillies ou ré-imaginées, l'àme d'un Maghreb unifié et de notre passé" (1982).

Plus peut-être qu'aucun autre cinéaste des années 70, Djebar parvient dans son second film à véritablement illustrer l'idée de Godard selon laquelle, devant toutes les manipulations de l'image, seule la bande sonore peut témoigner d'une vérité. Dans La Zerda ou les chants de l'oubli, c'est comme si la polyphonie des voix utilisées répondait à une séquence centrale du film accompagnée de ce commentaire: "Que restera-t-il de ce siècle excepté cette image" (1982) [on voit un officier français se faire cirer ses bottes par des enfants algériens]. 
Dans une des séquences les plus réussies du film (Chant de l'insoumission), la bande son mélange dans une interaction sans précédent, une voix chantant dans le registre des opéras occidentaux classiques et une autre s'exprimant dans celui des chansons populaires algériennes. Toutes deux chantent l'Emir Abdelkader et l'Algérie, leur duo cumule dans un crescendo quasi hystérique. Puis deux autres voix off parlant l'une en arabe, l'autre en français, precèdent de la même maniére jusqu'à un climax créant une véritable musique. Cette utilisation magistrale de la texture des voix et de la bande son intervient à un moment du film où l'image, destituée de tous ses pouvoirs narratifs cinématographiques habituels, est réduite à sa plus simple expression et pour ainsi dire paralysée dans son essence même (le cinéma étant par définition mouvement, qu'on songe au mot américain "movies"). C'est l'immobilité de photographies filmées qui fige alors l'écran.

\section{Incidence du cinéma sur les romans de Djebar}

Loin de ne constituer qu'un accident de parcours dans la carrière d'Assia Djebar, ces deux films ont en fait constitué une étape importante, une étape-clé de son évolution d'artiste. Lorsque treize ans après Les alouettes naives (1967) sort Femmes d'Alger dans leurs appartements (1980), il n'est point besoin d'une analyse détaillée pour remarquer immédiatement la filiation avec ses expériences cinématographiques. II s'agit là d'un titre hautement visuel puisqu'inspiré d'un tableau de Delacroix (1834) et la forme du récit adoptée est différente, morcelée en nouvelles (une nouveauté pour l'écrivain). Cette structure semble tout droit inspirée de l'éclatement cinématographique - composition en séquences divergentes - mais avec un fil conducteur que l'on trouvait dans La nouba des femmes du mont Chenoua.

Deux œuvres en particulier témoignent de l'influence majeure de ses réalisations cinématographiques sur son œuvre écrite, car chez Djebar, le passage de la plume à la caméra est suivi d'un retour de la caméra à la plume. C'est d'abord L'amour, la fantasia (1985) où dês la préface, Jean Berque signale l'originalité des matériaux de composition: "celle qui, dès les premières subversions de la conquête, s'était étalée en correspondances, mémoires, archives, envahit le public en assiégeant le privé, investit la quotidienneté par le journal, s'offre comme outil littéraire" (Berque, 1995:7). Le roman de Djebar est en effet un complexe assemblage de textes documentaires et de leurs commentaires, de récits historiques, d'entretiens et de morceaux autobiographiques ainsi que le souligne Denise Brahimi dans sa post-face intitulée "De la 'terre d'Afrique' à la contrée du dénuement": 
... ce qui chez la romancière entraîne une tentative multiple, ingénieuse et solidement structurée, pour dire la diversité du rapport qu'elle entretient avec son pays.

Elle le situe à la fois hors d'elle-mème, dans une histoire tantôt lointaine tantôt proche, et en elle-mème, dans une autobiographie déclarée (Brahimi, 1995:261)

\section{L'amour la fantasia}

Cet assemblage rappelle tout à fait la composition de son premier film. A quelques différences près - l'héroïne est cette fois-ci complètement identifiée avec l'écrivain dans un texte confidentiel rédigé à la première personne. (Mais est-ce réellement une différence? Les deux œuvres présentent une singulière similarité pour qui a vu des photographies de l'écrivain prises à l'époque du tournage de La nouba des femmes du mont Chenoua, la ressemblance physique entre elle et son actrice est assez gémellaire). Toutes les scènes se situant dans les grottes et qui dans le film sont traitées sur le mode lyrique et épique, sont ré-exploitées dans L'amour la fantasia mais dans une toute autre veine. Sur le plateau d'el Kantara, la tribu des Ouled Riah ne se résigne pas à remettre ses armes à l'armée de Pélissier, elle se cache dans des grottes avec femmes, enfants et bétails et se laisse enfumer, asphyxier et brûler plutôt que de se rendre aux Français (Djebar, 1985:91-97). Le drame nous est conté dans un style très réaliste où les détails graphiques contribuent à restituer le massacre dans toute son horreur. Par ailleurs, plusieurs voix de témoins se font entendre au cours du récit qui font écho aux interviews des paysannes combattantes du film. De plus, la troisième partie du texte est subdivisée en cinq mouvements qui nous renvoient à la composition musicale déjà évoquée à propos de La nouba des femmes du mont Chenoua. Enfin, le roman se clôt sur une partie répondant au "Khlass" qui concluait le film et intitulée dans le texte Tzarl'-rit (final), suivi de deux définitions:

'Tzart-rit':

- pousser des cris de joie en se

frappant les levres avec les mains

(Femmes)

- dictionnaire arabe-français

Beaussier

- crier, vociférer (les femmes,

quand quelque malheur leur arrive)

- dictionnaire arabe-français

Kazimirski (251) 
Ajoutons pour clore l'énumération de ces ressemblances les plus évidentes que cette conclusion est suivie d'une page de commentaires rédigée par Djebar et titrée "Air de Nay" (259).

Mais l'impact de l'œuvre cinématographique sur l'œuvre écrite est aussi sensible à un niveau plus conceptuel dans ce que l'on pourrait appeler une expérience de fusionnement des deux types d'écriture. Celle-ci se ressent par exemple dans la tentative pour lier certains chapitres comme on lie au cinéma deux séquences par une répétition d'image. Ainsi, le premier chapitre se terminant par les mots "... je suis partie à l'aube" (17), le second commence-t-il par la répétition du mot/photogramme "Aube de ce 13 juin $1830 \ldots$..." (18). Ce procédé n'est pas employé systématiquement mais se retrouve à plusieurs endroits du texte, on note ainsi: "... un étrange combat de femmes" (27), suivi à l'ouverture du chapitre suivant par "Le combat de Staouéli se déroule ..." (Djebar, 1985:28). Ou encore "... quelque soudaine explosion" (Djebar, 1985:42) et "Explosion du Fort l'Empereur, ..." (43), "... autant dire s'aimait ouvertement" (53), et "Ouverte la Ville plutôt que prise" (54), etc., etc. Bien que ce procédé s'arrète à la fin de la premiêre partie il constitue sans aucun doute une des transpositions les plus réussies d'une technique cinématographique de montage à laquelle Djebar s'était essayée avec beaucoup moins de succès dans son premier film que dans L'amour, la fantasia. Là où les séquences du film restaient désespérément hétérogénes (ceci pouvant, il est vrai, ètre vu comme un signe de modernité d'écriture), la liaison dans le roman entre les textes autobiographiques, les documents historiques et les récits de témoignages s'opére sans heurts dans une fusion/lissage du style qui parvient à allier les matériaux de narration les plus divers.

De l'utilisation si originale de la bande son des deux films on retrouve la résonance tout au long du roman où de nombreux chapitres s'intitulent "Voix". En plusieurs endroits du texte, l'auteur s'attarde à la description de certaines d'entre elles. Par exemple, celle qu'elle nomme "la première réalité-femme": "la voix, elle, entre en chacun comme un parfum, une gorgée d'eau pour gosier d'assoiffé; et lorsqu'elle se goûte, elle devient plaisir pour plusieurs, simultanément : secrète jouissance polygame ..." (208). Ou, lorsqu'elle évoque les voix qui l'entourent dans sa retraire parisienne:

je m'oublie des heures à percevoir des voix sans visages, des bribes de dialogues, des brisures de récits, tout un balbutiement, un buissonnement de bruits détachés du magma des faces, délivrés de l'inquisition des regards (248). 


\section{Loin de Médine}

En ce qui concerne Loin de Médine ( 1991), l'agencement du roman défini par l'écrivain elle-même reflète encore une fois la composition éclatée des films: "J'ai appelé "roman" cet ensemble de récits, de scènes, de visions parfois, qu'a nourri en moi la lecture de quelques historiens des deux ou trois premiers siècles de l'Islam" (Djebar, 1991:5). Plus encore que dans L'amour, la fantasia, la continuité de l'inspiration cinématographique est ici évidente. Avec La Zerda ou les chants de loubli Djebar avait abandonné la fiction pour la réintroduire dans les films d'archives, dans Loin de Médine, les textes des historiens lui sont prétexte à développer sa narration.

L'importance de la voix dans ce livre est comme démultipliée. Des segments de texte réapparaissent de manière récurrente et sont intitulés "Voix". D'autres titres référent à la parole ou au son, "Point d'orgue" (269), "Parole vive" (281), "Celle qui préserve la parole vive" (326), "Parole plurielle, parole duelle" (337). Mais c'est l'essence elle-même du roman tout entier qui repose sur la voix. Deux épisodes en rendent bien compte. Le plus exemplaire est le récit concernant la chanteuse de satire. Poétesse au nom inconnu elle est condamnée par le chef des guerriers musulmans Mohadjir ibn Ommeyya à la prise de la forteresse de Beni Kinda. L'artiste lyrique, dénoncée au chef des armées, a les mains coupées par ses soldats mais surtout les dents arrachées pour ne plus pouvoir chanter. La voix, source de vérité est ainsi enrayée : "Tandis qu'elle défaille, elle comprend: sa voix va siffler, sa voix va grincer, sa voix ne va pas chanter tout ce qui, à l'instant même, se presse en son coeur en strophes toutes prêtes, aussi chaudes que le sang qu'elle crache" (135).

L'autre anecdote se situe dans le chapitre intitulé "La combattante". Elle peut en fait servir d'illustration au concept même de Loin de Médine. Une femme mecquoise, Oum Hakim, relate ses souvenirs concernant le dernier discours de Mohammed lors du pêlerinage de l'an 10, un mois avant sa mort. Le récit est mis en scène d'une manière mettant bien encore l'accent sur la primauté du son, de la voix, par rapport à l'image:

Voici que, deux ans après, elle suivait sa voix à la trace parmi les méandres de cette foule impressionnante et elle éprouvait le besoin de le voir. Contempler Mohammed. (...) Elle comprit que Mohammed avait choisi de se poster sur cette éminence pour que sa voix porte ... (162).

Mais l'image se brouille. Ce qui compte, c'est le message porté par la parole du Prophète. 
Soudain l'image du Prophéte sur sa chamelle rousse se brouilla devant Oum Hakim, tremblota, se dilata comme dans un halo. Elle ne sut si c'était l'excés de chaleur ou la lumière aveuglante et blanchie. Elle fut propulsée sur le côté, et un long chant profus, scandé doublement, éclata: Bilal reprenait la question du Messager. Oum Hakim percevait, comme voguant à travers d'autres sphères, la voix aérienne de Mohammed. A la phrase reprise amplifiée, par la voix fortement timbrée de Bilal, la foule autour de Oum Hakim, répondait une première fois (162-163).

\section{Conclusion: l'image ef la voix}

Pendant tout le reste de la scène, l'héroïne n'entend plus que la voix du Prophète ; elle ne perçoit le reflet de son image que dans le regard des autres. Double tàche de la romancière et des témoins de I'Islam: rapporter le message et la vie du Prophète à travers la transmission des souvenirs de son discours et des récits des témoins de son existence. Denise Brahimi saisit bien l'importance de cette sonorité des romans lorsqu'elle déclare: "Le moment vient où l'effet immédiatement bouleversant de ces voix fait place à la perception d'un étrange silence qui les entoure, et dans lequel elles font écho d'autant plus poignant qu'il semble déjà lointain" (Brahimi, 1985:265).

Jamais peut-ètre, un écrivain n'avait à ce point lié étroitement sa pratique cinématographique à sa technique romanesque. Le travail de Djebar s'avère particulièrement réussi dans un domaine comme dans l'autre. Aux balbutiements maladroits de La nouba des femmes du mont Chenoua, inspiration gauche de certaines théories cinématographiques d'avant-garde des années 70 , fait suite la pleine réussite de La Zerda ou les chants de l'oubli. Les recherches de Duras ne peuvent qu'aboutir au nihilisme de cette réplique du Camion (1976) "Que le monde aille à sa perte, c'est la seule solution", qu'aux lieux détruits, vides de tout personnage de Son nom de Venise dans Calcutta désert (1975). Djebar, elle, recentre le matériau filmique sur des images d'archives plutôt que d'utiliser une image exsangue, anémiée, et retravaille la bande sonore dans la musicalité polyphonique et poétique. Djebar porte à un point de richesse créatrice jamais atteint auparavant la théorie de la prédominance du son sur l'image sans détruire cette dernière mais en la remettant à sa place, ni minimisée, ni surévaluée. Pour reprendre l'expression de Godard: "Juste une image / Une image juste" (Tout va bien:1972).

Pour ce qui retourne de l'écriture, Djebar renouvelle son style sous l'influence de son expérience cinématographique, pour le porter à sa plus parfaite expression dans l'écriture de Loin de Médine. Jamais peut- 
être un écrivain musulman n'avait mieux fait s'épouser le fond et la forme, la voix littéraire et le message religieux. Dans Loin de Médine c'est la voix de celui dont toute représentation picturale est un interdit sacrilège qui nous est rendue. La ferveur du son redonne à l'image condamnée une virginité, une profondeur que l'ouïe nous restitue bien mieux que l'oeil.

\section{Références}

Armes, Roy. 1996. Dictionnaire des cinéastes du Maghreb. Tome 2. Paris : ATM

Brahimi, Denise. 1995. De la 'terre d'Afrique' à la contrée du dénuement. Post-face à

L'amour la fantasia d'Assia Djebar, édition marocaine. Casablanca : EDDIF. p. 261-268.

Berques, Jacques. 1995. La mémoire longue d'une romancière maghrébine. Préface

à L'amour la fantasia d'Assia Djebar, édition marocaine. Casblanca : EDDIF. $p$. 7-9.

Djebar, Assia. 1962. Les enfants du nouveau monde. Paris : Julliard.

Djebar, Assia. 1967. Les alouettes naives. Paris : Julliard.

Djebar, Assia.1980. Fermmes d'Alger dans leur appartement. Paris : Des femmes.

Djebar, Assia. 1985. L'Amour, la fantasia. Paris : Lattès.

Djebar, Assia. 1991. Loin de Médine. Paris : Albin Michel.

Films

Djebar, Assia. 1978. La nouba des femmes du mont Chenoua. RTA

Djebar, Assia.1982. La zerda ou les chants de l'oubli. RTA.

Duras, Marguerite. 1969. La Musica.

Duras, Marguerite. 1969 Détruire, dit-elle.

Duras, Marguerite. 1975. India Song.

Duras, Marguerite. 1975. Son nom de Venise dans, Calcutta désert.

Duras, Marguerite. 1976. Le camion.

Godard, Jean-Luc. 1970. Vent d'est, (sous le nom Groupe Dziga Vertov)

Godard, Jean-Luc. 1972. Tout va bien.

Key concepts:

Assia Djebar

North African woman writer

novel-film: reciprocal influence

writer-filmmaker; dual career

Kernbegrippe:

Assia Djebar

roman-film: wedersydse beïnvloeding

romanskrywer-rolprentvervaardiger; tweeledige loopbaan

vroueskrywer uit Noord-Afrika 
